\title{
Machine learning to differentiate small round cell malignant tumors and non-small round cell malignant tumors of the nasal and paranasal sinuses using apparent diffusion coefficient values
}

\author{
Chen Chen ${ }^{1}$. Yuhui Qin ${ }^{1} \cdot$ Haotian Chen ${ }^{1}$ Junying Cheng ${ }^{2} \cdot$ Bo He ${ }^{1}$ Yixuan Wan ${ }^{1}$. Dongyong Zhu ${ }^{1}$ Fabao Gao ${ }^{1}$. \\ Xiaoyue Zhou ${ }^{3}$
}

Received: 27 July 2021 / Revised: 10 November 2021 / Accepted: 14 November 2021 / Published online: 14 January 2022

(c) The Author(s) 2022

\begin{abstract}
Objective We used radiomics feature-based machine learning classifiers of apparent diffusion coefficient (ADC) maps to differentiate small round cell malignant tumors (SRCMTs) and non-SRCMTs of the nasal and paranasal sinuses.

Materials A total of 267 features were extracted from each region of interest (ROI). Datasets were randomized into two sets, a training set $(\sim 70 \%)$ and a test set $(\sim 30 \%)$. We performed dimensional reductions using the Pearson correlation coefficient and feature selection analyses (analysis of variance [ANOVA], relief, recursive feature elimination [RFE]) and classifications using 10 machine learning classifiers. Results were evaluated with a leave-one-out cross-validation analysis.

Results We compared the AUC for all the pipelines in the validation dataset using FeAture Explorer (FAE) software. The pipeline using RFE feature selection and Gaussian process classifier yielded the highest AUCs with ten features. When the "one-standard error" rule was used, FAE produced a simpler model with eight features, including Perc.01\%, Perc.10\%, Perc.90\%, Perc.99\%, S(1,0) SumAverg, S(5,5) AngScMom, S(5,5) Correlat, and WavEnLH_s-2. The AUCs of the training, validation, and test datasets achieved 0.995, 0.902, and 0.710, respectively. For ANOVA, the pipeline with the auto-encoder classifier yielded the highest AUC using only one feature, Perc.10\% (training/validation/test datasets: 0.886/0.895/0.809, respectively). For the relief, the AUCs of the training, validation, and test datasets that used the LRLasso classifier using five features (Perc.01\%, Perc.10\%, S(4,4) Correlat, S(5,0) SumAverg, S(5,0) Contrast) were 0.892, 0.886, and 0.787, respectively. Compared with the RFE and relief, the results of all algorithms of ANOVA feature selection were more stable with the AUC values higher than 0.800 .

Conclusions We demonstrated the feasibility of combining artificial intelligence with the radiomics from ADC values in the differential diagnosis of SRCMTs and non-SRCMTs and the potential of this non-invasive approach for clinical applications. Key Points

- The parameter with the best diagnostic performance in differentiating SRCMTs from non-SRCMTs was the Perc.10\% ADC value.

- Results of all the algorithms of ANOVA feature selection were more stable and the AUCs were higher than 0.800, as compared with RFE and relief.

- The pipeline using RFE feature selection and Gaussian process classifier yielded the highest AUC.
\end{abstract}

Keywords Apparent diffusion coefficient $\cdot$ Radiomics $\cdot$ Machine learning $\cdot$ Neoplasms

Abbreviations
$\mathrm{AB} \quad$ Ada-boost
$\mathrm{AE}$

Fabao Gao

gaofabao@wchscu.cn

1 Molecular Imaging Laboratory, Department of Radiology, West China Hospital, Sichuan University, 37 Guoxue Road, Chengdu, Sichuan 610041, People's Republic of China

$\begin{array}{ll}\text { AGS } & \text { Absolute gradient statistics } \\ \text { ANOVA } & \text { Analysis of variance } \\ \text { ARM } & \text { Auto-regressive model } \\ \text { DT } & \text { Decision tree }\end{array}$

2 Department of MRI, the First Affiliated Hospital of Zhengzhou University, Zhengzhou, People's Republic of China

3 MR Collaboration, Siemens Healthineers Ltd., Shanghai, People's Republic of China 


$\begin{array}{ll}\text { GLCM } & \text { Gray-level co-occurrence matrix } \\ \text { GLRLM } & \text { Gray-level run-length matrix } \\ \text { GP } & \text { Gaussian process } \\ \text { LDA } & \text { Linear discriminant analysis } \\ \text { LR } & \text { Logistic regression } \\ \text { LRLasso } & \text { Logistic regression via Lasso } \\ \text { ML } & \text { Machine learning } \\ \text { NB } & \text { Naive Bayes } \\ \text { PCC } & \text { Pearson correlation coefficient } \\ \text { RF } & \text { Random forests } \\ \text { RFE } & \text { Recursive feature elimination } \\ \text { SMOTE } & \text { Synthetic minority oversampling technique } \\ \text { SRCMTs } & \text { Small round cell malignant tumors } \\ \text { SVM } & \text { Support vector machine } \\ \text { TA } & \text { Texture analysis } \\ \text { WAV } & \text { Wavelet transform }\end{array}$

\section{Introduction}

Malignant tumors of the nasal and paranasal sinuses are rare, comprising less than $1 \%$ of all malignancies and about $3 \%$ of head and neck malignancies [1,2]. This tumor group includes small round cell malignant tumors (SRCMTs) and non-SRCMTs. SRCMTs constitute a specific group of malignancies in the nasal and paranasal sinuses based on neuroectodermal differentiation, soft tissue differentiation, and hematopoietic differentiation. Rhabdomyosarcoma (RMS), malignant melanoma (MM), olfactory neuroblastoma (ONB), neuroendocrine carcinoma (NEC), and lymphoma are included in this group. Non-SRCMTs constitute another common group of malignant tumors in the nasal and paranasal sinuses based on epithelial differentiation and include squamous cell carcinoma (SCC) and adenoid cystic carcinoma (ACC) [3]. Distinguishing these two groups is elemental because some are managed primarily with radiation, whereas others are managed solely with chemotherapy. Still others are managed with conservative medical therapy, local surgery, exenterative surgery, and multimodal therapy, indicating that therapeutic decisions, surgical planning, and prognoses are different for the various tumor types and management strategies [4].

Varying according to the pathology and cellularity of the tissue because of the limited diffusion of water molecules, apparent diffusion coefficient (ADC) values have been used to discriminate malignant from benign nasal and paranasal sinus tumors and to differentiate various histopathologic types of malignant sinonasal tumors [5-10]. However, conventional magnetic resonance imaging (MRI) has limitations of its own when differentiating between SRCMTs and non-SRCMTs. Under the circumstances, as texture analysis (TA) techniques, by using mathematically defined features[11], can analyze pixel distributions, intensities, and dependencies, it can provide a wealth of information beyond what can be seen with the human eye and thus can be used to characterize SRCMTs and non-SRCMTs, quantitatively.

As a branch of artificial intelligence, machine learning (ML) includes various algorithms that can enhance diagnosis, treatments, and follow-up results in neuro-oncology medicine by analyzing huge complex datasets $[12,13]$. More importantly, not depending on user experience, ML is more objective than other conventional analyses and has good repeatability. To achieve the optimal predictive ability and clinical utility, in the present study, we compared three feature selection methods and an array of ML algorithms. To our knowledge, no studies using TA and ML for differentiating sinonasal SRCMTs from non-SRCMTs have been reported. To bridge this gap, this retrospective study was intended to evaluate the potential value of the ML-based ADC texture analysis for distinguishing SRCMTs from nonSRCMTs by using various state-of-the-art ML algorithms.

\section{Materials and methods}

\section{Patients}

We used the surgical pathology database from January 1, 2018, to November 1, 2020, at our hospital. Exclusion criteria were (1) patients who received treatments before surgery and (2) inadequate image quality. All the methods were performed in accordance with the relevant guidelines and regulations, and informed consent was waived. This study was approved by the Institutional Ethics Review Committee of our hospital.

\section{Image acquisition}

Patients were examined with a 3-T MR scanner (Siemens Skyra) with standard head coil. MRI scan protocols included the following: axial T2WI (TR/TE $=5000 / 117$ $\mathrm{ms}$, matrix $=256 \times 256$, field of view $=24 \times 24 \mathrm{~cm}$, thickness $=5 \mathrm{~mm}$ and intersection gap $=1 \mathrm{~mm}$ ); axial DWI (spin echo-echo planar imaging) $\left(\mathrm{b}=0\right.$ and $1000 \mathrm{~s} / \mathrm{mm}^{2}$, $\mathrm{TR} / \mathrm{TE}=3200 / 70 \mathrm{~ms}$, matrix $=160 \times 160$, flip angle $90^{\circ}$, field of view $=24 \times 24 \mathrm{~cm}$, thickness $=5 \mathrm{~mm}$, intersection gap $=1 \mathrm{~mm}$ ).

\section{Textural feature extractions}

MaZda v. 4.7 software (The Technical University of Lodz, Institute of Electronics, http://www.eletel.p. lodz.pl/mazda/) was used for the analyses. We used the limitation of dynamics to $\mu \pm 3 \delta$ ( $\mu$ : mean gray-level value, $\delta$ : standard deviation) [14] to achieve reliable results regarding MRI texture classifications. Regions 
of interest (ROIs) were drawn on ADC images. The largest lay was selected using a T2WI image reference. Two physicians with more than 10 years of experience delineated the ROIs manually along the lesion edges, and the lesion was filled in with a red marker, excluding various necrotic and cystic regions (Fig. 1). In total, 267 feature values and corresponding histogram maps were extracted for each ROI. The number of radiomics features based on feature classes is presented in Table 1, including (i) nine histogram features based on the pixel counts in an image with a specific gray-level value [15], (ii) 220 Gy-level co-occurrence matrix (GLCM) features based on the extracted statistical information about the distribution of pixel pairs [16], (iii) 20 Gy-level runlength matrix (GLRLM) features obtained by searching the image for runs having the same gray-level value in a pre-defined direction [17], (iv) 5 auto-regressive model (ARM) features based on the weights associated with four neighboring pixels and the variance of the minimized prediction error, (v) 8 wavelet transform (WAV) features on texture frequency components extracted from the energies computed within the channels [18], and (vi) 5 absolute gradient statistics (AGS) features based on the spatial variation of gray-level values across the image [15]. Multiple GLCMs were computed into the $0^{\circ}, 45^{\circ}$, $90^{\circ}, 135^{\circ}$, and $z$-axis directions and $1,2,3$, and 4 pixels. Multiple GLRLMs were computed along four different angles (horizontal, vertical, diagonal 45, and diagonal 135).

\section{Feature selections}

Computer-generated random datasets were used to assign $70 \%$ of the datasets to the training set and the rest $(30 \%)$ of the datasets to the independent test set. FeAture Explorer software (FAE; V 0.3.6) was developed using the Python programming language (3.7.6) (https:// github.com/salan668/FAE). First, the synthetic minority oversampling technique (SMOTE) was used to balance the training dataset. This method worked by taking each minority class sample, introducing synthetic examples along the line segments and joining any or all of the nearest $k$ minority class neighbors. The neighboring points were randomly chosen depending on the amount of oversampling required. The dataset was normalized using $Z$-score normalization, which subtracted the mean value and divided the standard deviation for each feature. Second, a Pearson correlation coefficient (PCC) was used for each pair of features to reduce the dimensions of the row space of the feature matrix [19]. If the PCC
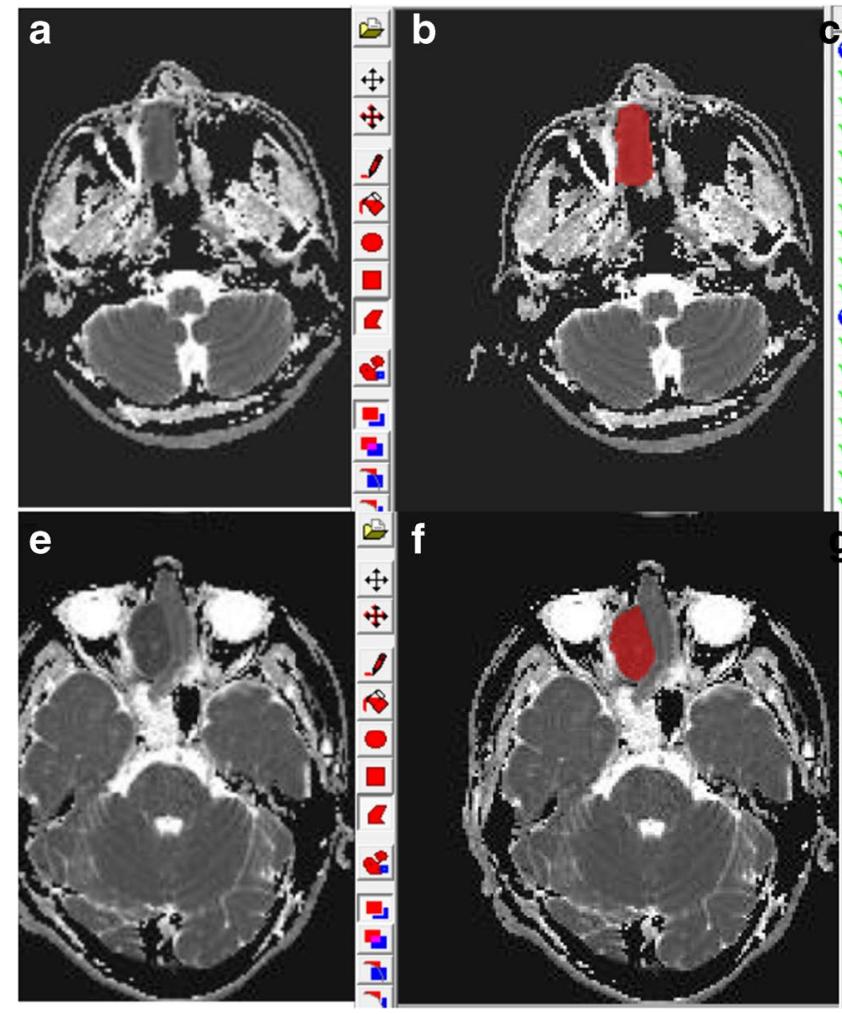

Fig. 1 a shows axial ADC of a 36-year-old male patient with SCC. b Corresponding ROI, (c) parts of 267 feature values, and (d) histogram maps are shown. (e) shows axial ADC of a 44-year-old male patient

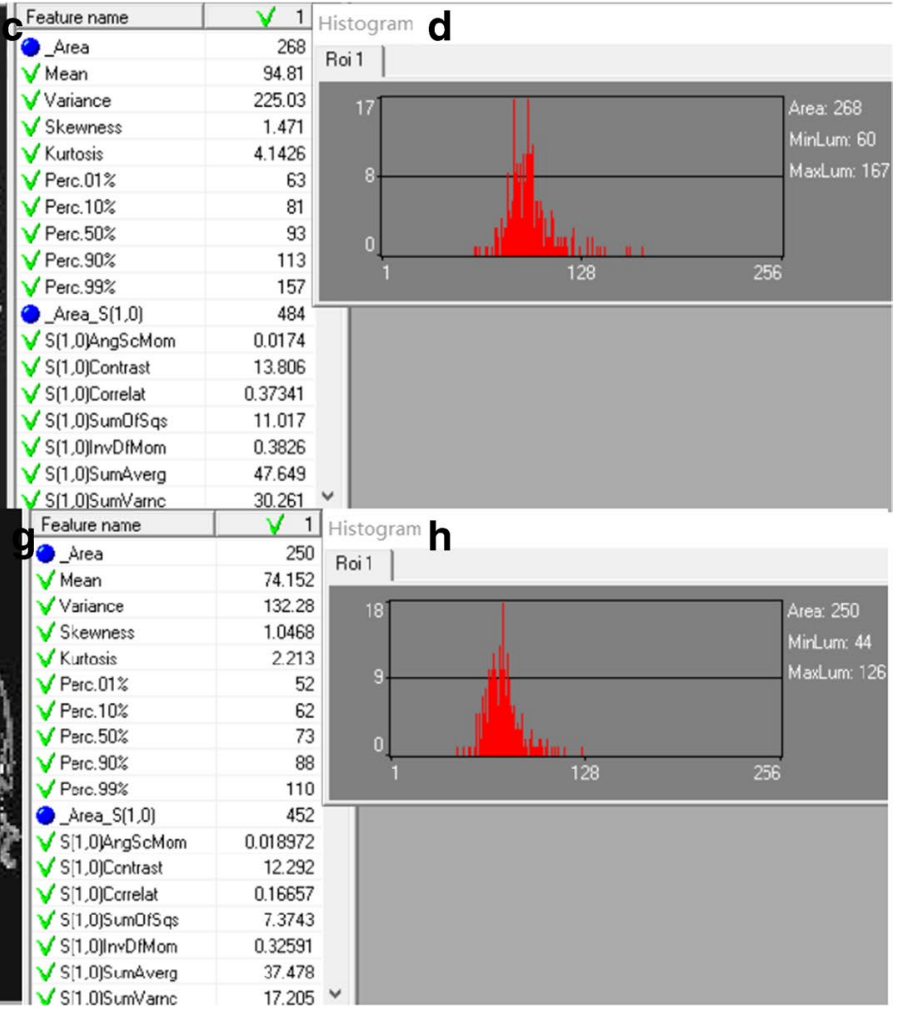

with ONB. (f) Corresponding ROI, (g) some of 267 feature values, and (h) histogram maps are shown 
Table 1 Texture analysis methods and the corresponding texture features

\begin{tabular}{|c|c|}
\hline Method & Texture feature parameters \\
\hline Histogram (9) & Mean, variance, skewness, kurtosis, and percentiles $(1 \%, 10 \%, 50 \%, 90 \%$, and $99 \%)$ \\
\hline Gray-level co-occurrence matrix (GLCM) (220) & $\begin{array}{l}\text { Angular second moment (AngScMom), contrast, inverse different moment (IDM), entropy } \\
\text { (Ent), correlation (Correlat), sum of squares (SumOfSqs), sum average (SumAverg), sum } \\
\text { variance (SumVarnc), sum entropy (SumEntrp), difference variance (DifVarnc), difference } \\
\text { entropy (DifEntrp) along the } 0^{\circ}, 45^{\circ}, 90^{\circ}, 135^{\circ} \text {, and } z \text {-axis directions and } 1,2,3 \text {, and } 4 \\
\text { pixels }\end{array}$ \\
\hline Gray-level run-length matrix (GLRLM) (20) & $\begin{array}{l}\text { Run-length nonuniformity (RLNonUni), gray-level nonuniformity (GLevNonU), long run } \\
\text { emphasis (LngREmph), short run emphasis (ShrtREmp), fraction of image in runs (Frac- } \\
\text { tion) of four different angels (horizontal, vertical, diagonal 45, and digonal135) }\end{array}$ \\
\hline Auto-regressive model (ARM) (5) & Teta1, Teta2, Teta3, Teta4, Sigma \\
\hline Wavelet transform (WAV) (8) & $\begin{array}{l}\text { Energy computed from the low-low frequency band within the first image scale } \\
\text { (WavEnLL_s-1), WavEnLH_s-1, WavEnHL_s-1, WavEnHH_s-1, WavEnLL_s-2, } \\
\text { WavEnLH_s-2, WavEnHL_s-2, WavEnHH_s-2 }\end{array}$ \\
\hline Absolute gradient statistics (AGS) (5) & $\begin{array}{l}\text { Absolute gradient mean (GrMean), variance (GrVariance), skewness (GrSkewness), kurtosis } \\
\text { (GrKurtosis), nonzeros (GrNonZeros) }\end{array}$ \\
\hline
\end{tabular}

was above 0.99 , one of features was randomly removed. Lastly, the analysis of variance (ANOVA), relief [20], and recursive feature elimination (RFE) were utilized for feature selections. ANOVA is a common method that explores the significant features corresponding to the labels. Relief selects sub-datasets and finds relative features according to the recursive labels. RFE is intended to select features based on a classifier by recursively considering a smaller set of features. The range of the number of features was set from 1 to 20 .

\section{Classifications}

The classification performances were tested with $10 \mathrm{ML}$ algorithms based on Python code with scikit-learn library (https://scikit-learn.org/), including the support vector machine (SVM), linear discriminant analysis (LDA), autoencoder (AE), random forests (RF), logistic regression (LR), logistic regression via Lasso (LRLasso), ada-boost (AB), decision tree (DT), Gaussian process (GP), and naive Bayes (NB) (Table 2). SVM searches for an optimal separating

Table 2 The parameters of the algorithms

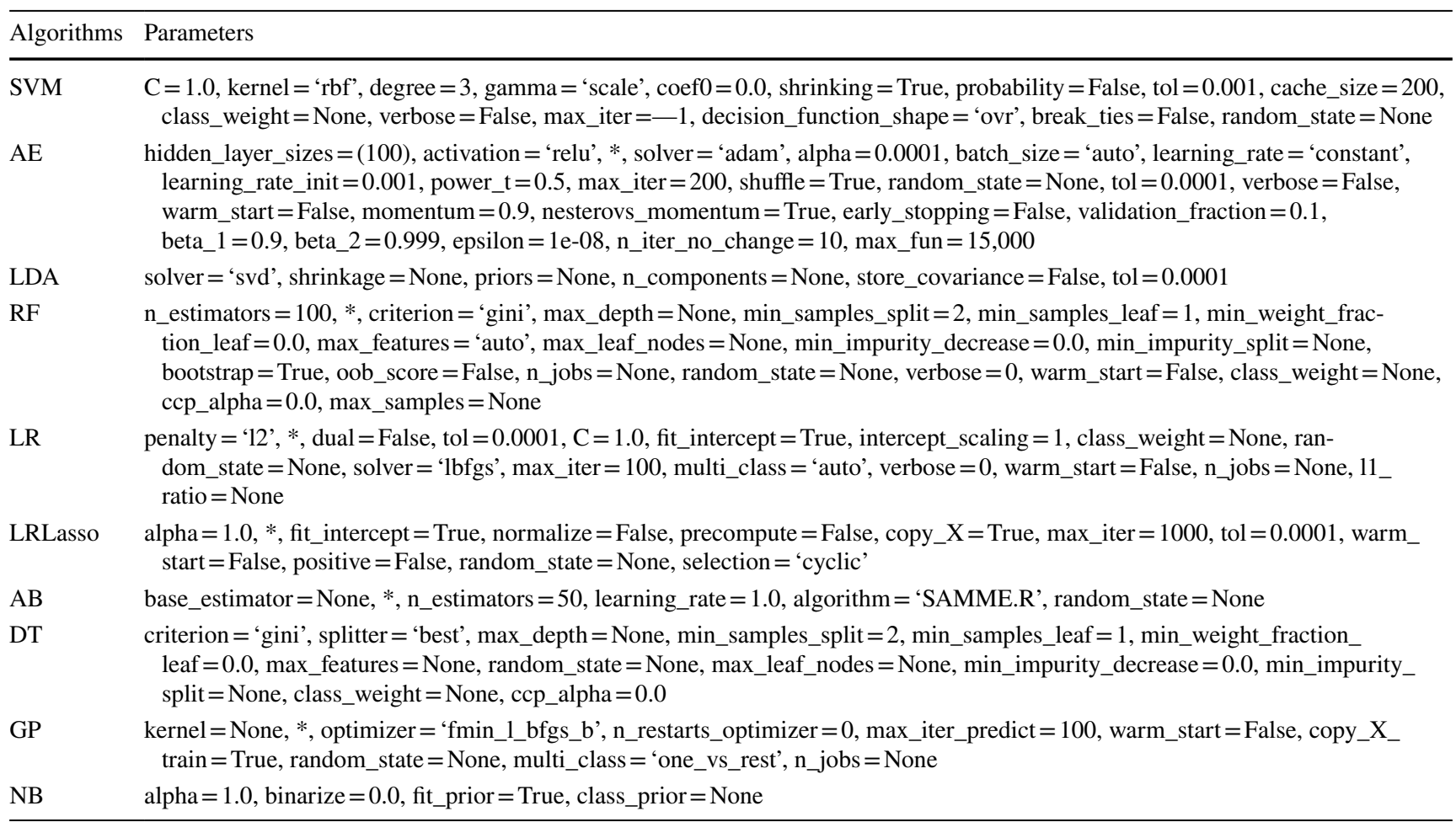


hyperplane between classes, which maximizes the margin. C stands for regularization parameter. The strength of the regularization is inversely proportional to C. AE classification is based on neural networks (NN), which is a network of highly interconnected processing units that process information by their dynamic state responses to external inputs. LDA with a linear decision boundary was generated by fitting class conditional densities to the data and using Bayes' rule. Solver uses singular value decomposition recommended for data with a large number of features. RF consists of a large number of individual decision trees that operate as an ensemble. Each individual tree outputs a class prediction and the class with the most votes represents the model's prediction. The number of trees in the forest was 100. In most cases, the larger the number, the better the performance. LR explains the relationship between one dependent binary variable and one or more independent variables regressing for the probability of a categorical outcome using a logistic function. Lasso-LR is able to get a better model which can do high-dimensional statistics. Alpha is equivalent to an ordinary least square with defaults to 1.0. $\mathrm{AB}$ generates $\mathrm{H}$ hypotheses through an ensemble of learning algorithms. The output of the learning algorithms is incorporated into a weighted sum that represents the final output of the boosted classifier. DTs of supported criteria are "gini" for the Gini impurity and "entropy" for the information gain. GP was based upon Laplace approximation. The kernel was none, specifying the covariance function of the GP. NB applies Bayes' theorem with the naive assumption of conditional independence between the features. Setting alpha $=1.0$ is called Laplace smoothing.

\section{Evaluations}

The results were evaluated using a leave-one-out cross-validation (LOOCV) test. Using LOOCV, learning sets were created by taking all the samples except one that was used as the validation set. The accuracy, sensitivity, specificity, positive predictive value (PPV), and negative predictive value (NPV) were also calculated at a cutoff value that maximized the value of the Youden index. The area under the receiver operator characteristics curve (AUC) of the classification results was calculated for each tested condition (Fig. 2).

\section{Results}

Of the 168 consecutive patients with a pathologic diagnosis of SRCMT and non-SRCMT over a 2-year period between January 2018 and November 2020, 16 were excluded with poor-quality images on MRI ( 7 were excluded due to the visible artifacts from dental work, 2 due to motion artifacts, and 7 due to visible artifacts from the bone-air interface).
A total of 152 patients were finally selected for the study. There were 66 patients with SRCMT and 86 patients with non-SRCMT, specifically RMS $(n=14)$, lymphoma $(n=20)$, MM $(n=10)$, NEC $(n=14)$, ONB $(n=8), \operatorname{SCC}(n=62)$, and ACC $(n=24)$. There were 88 males and 64 females in the whole cohort. The mean age of all the patients was 54.28 years, ranging from 13 to 87 years. Seventy percent of the datasets were in the training set (106 patients; 46 with SRCMT and 60 with non-SRCMT) and 30\% in the independent test set (46 patients; 20 with SRCMT and 26 with non-SRCMT).

SMOTE was used to automatically generate 14 synthetic SRCMT samples in order to overcome the influence of imbalanced dataset on the classifier fitting. We compared the AUC of all the pipelines on the validation dataset with FAE. The pipeline using RFE feature selection and a GP classifier yielded the highest AUCs using ten features. When the "one-standard error" rule was used, FAE also produced a simpler model with eight features [21]. The ROC curves are shown in Fig. 3. The AUCs of the training, validation, and test datasets achieved $0.995,0.902$, and 0.710 , respectively. FAE-selected features were Perc.01\%, Perc.10\%, Perc.90\%, and Perc. $99 \%$ from the histogram; S(1,0) SumAverg, S(5,5) AngScMom, and $\mathrm{S}(5,5)$ Correlat from gray-level GLCM; and WavEnLH_s-2 from wavelets transform (WAV).

As for ANOVA, the pipeline using the AE classifier yielded the highest AUC using one feature with a "onestandard error" rule, as shown in Fig. 4. The AUCs of the training, validation, and test datasets achieved 0.886, 0.895, and 0.809 , respectively. The FAE-selected feature was Perc. 10\% from the histogram $(F=84.24, p<0.001)$.

As for relief, the pipeline using the LRLasso classifier yielded the highest AUC using five features. Features selected by FAE were Perc.10\% and Perc.01\% from histogram, and $S(4,4)$ Correlat, $S(5,0)$ SumAverg, and $S(5,0)$ Contrast from GLCM; weights were 1.09, 1.07, 0.86, 0.82, and 0.76 , respectively. When the "one-standard error" rule was used, FAE also produced a simpler model with only one feature; the ROC curves are shown in Fig. 5. The AUCs of the training, validation, and test datasets achieved 0.892 , 0.886 , and 0.787 , respectively. The feature selected from the histogram with FAE was Perc.10\%.

Using the RFE feature selection, the AUCs of the training, validation, and test datasets of the $10 \mathrm{ML}$ algorithms were greater than 0.640 (Fig. 6A). The optimal algorithm in the validation datasets was GP, whose AUC was 0.902. In all the algorithms, the AUCs of training datasets were better than those of validation datasets whose AUCs were greater than those of the test datasets.

Using ANOVA feature selection, the AUCs of the training, validation, and test datasets of ten machine learning algorithms were greater than $\sim 0.800$ (Fig. 6B). The optimal algorithm in the validation datasets was AE, whose AUC 
Fig. 2 A schematic diagram for the whole radiomics and machine learning pipeline

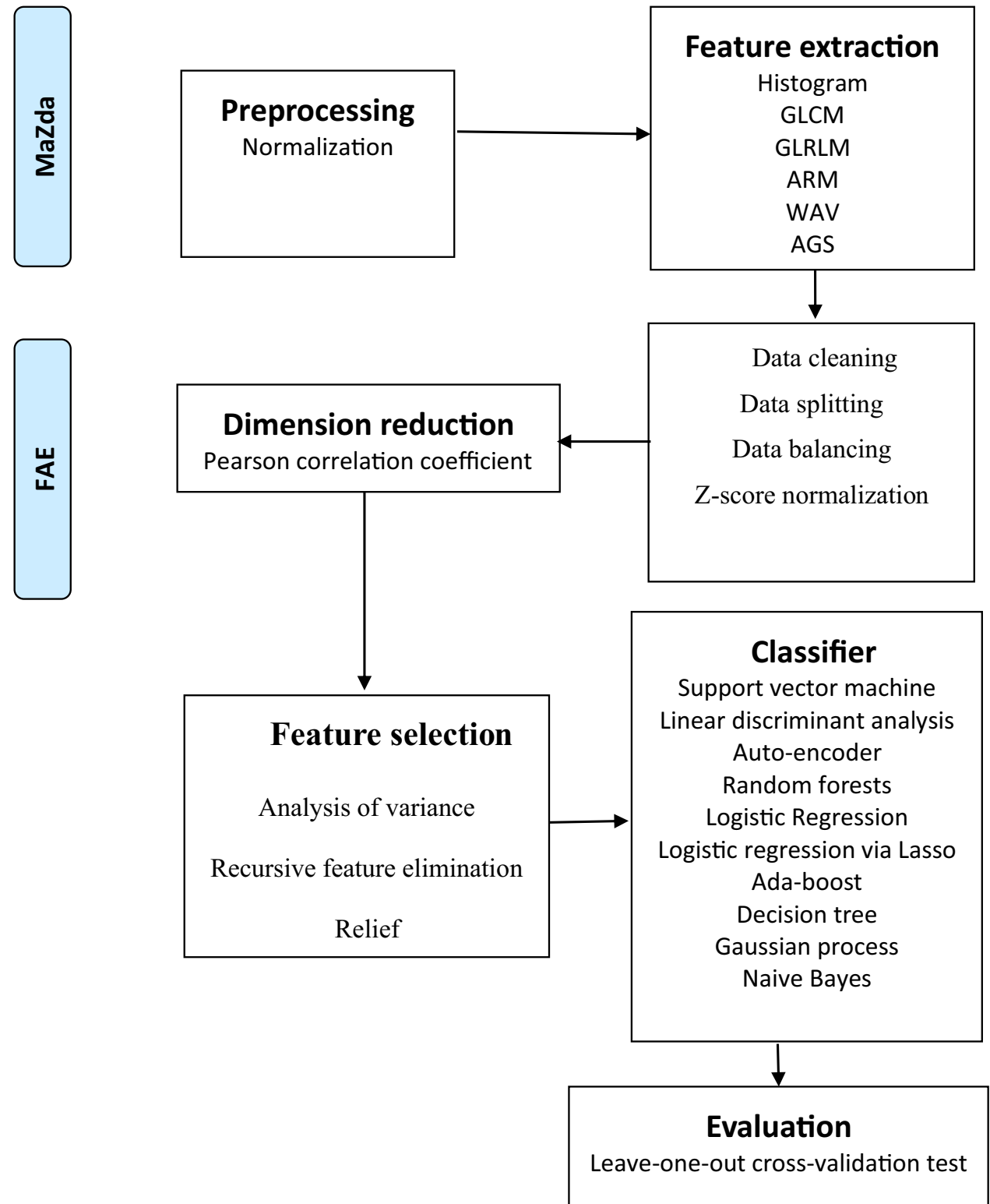

was 0.895. Compared with RFE and relief, the results of all the algorithms of ANOVA feature selection were more stable.

Using relief feature selection, the AUCs of the training, validation, and test datasets of nine $\mathrm{ML}$ algorithms were greater than $\sim 0.700$ except AE (Fig. 6C). The optimal algorithm in the validation datasets was LRLasso, whose AUC was 0.886 . In the nine algorithms except AE, the AUCs of training datasets were better than those of validation datasets, whose AUCs were greater than those of the test datasets.

In addition to the three feature selection methods, we also compared and listed the optimal AUCs of different ML classifications in the validation dataset (Table 3).

\section{Discussion}

This study investigated the potential value of the ADC texture analysis for distinguishing SRCMTs from non-SRCMTs by using various state-of-the-art ML algorithms. The key findings are as follows: (1) the pipeline using RFE feature selection and Gaussian process classifier yielded the highest AUC. (2) The parameter with the best diagnostic performance in differentiating SRCMTs from non-SRCMTs was the Perc.10\% ADC value. (3) Results of all the algorithms of ANOVA feature selection were more stable and the AUCs were higher than 0.800 , as compared with RFE and relief. (4) TA and ML appear to be the most useful in differentiating standard ADC images of maximum solid tumor 
a Receiver operating characteristic curve

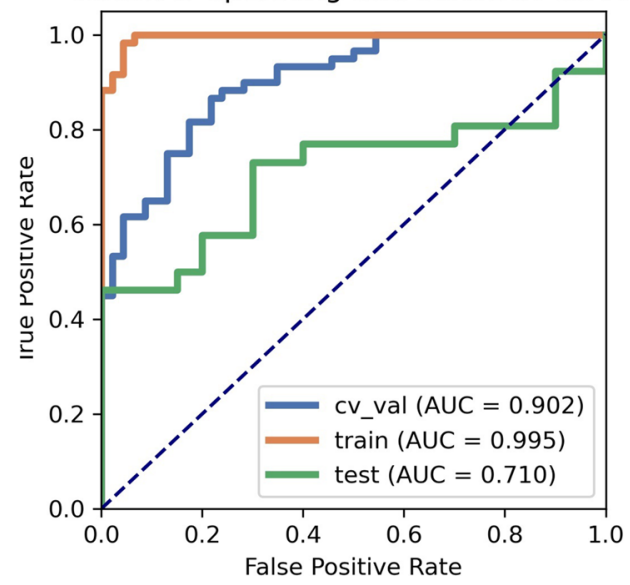

b

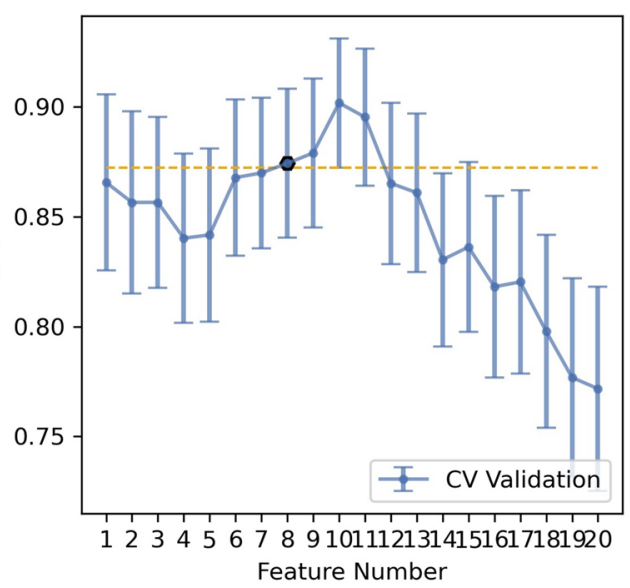

C

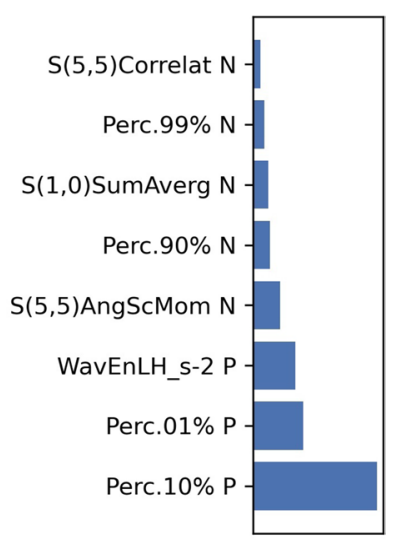

Fig. 3 Model performance generated using recursive feature elimination. a Receiver operating characteristic (ROC) curves of this model using different datasets. b FeAture Explorer (FAE) software sug- gested a candidate eight-feature model according to the "one-standard error" rule. $\mathbf{c}$ The contribution of features in the final model a

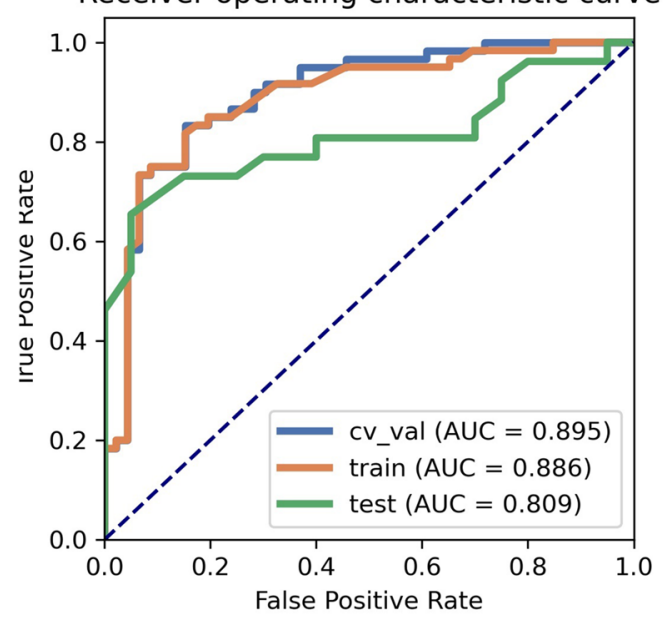

b

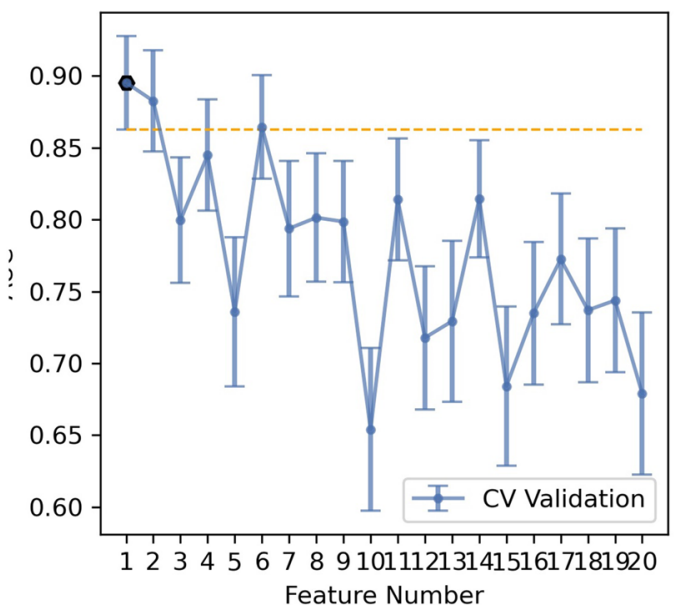

C

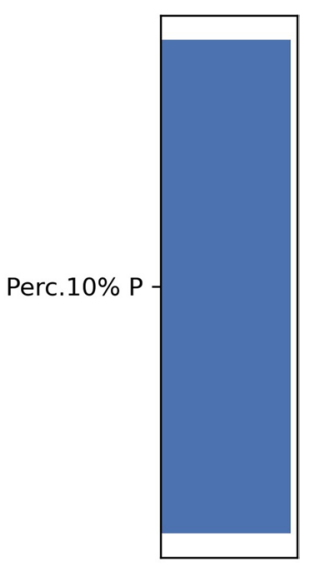

Fig. 4 Model performance generated using the analysis of variance (ANOVA). a Receiver operating characteristic (ROC) curves of this model using different datasets. b FeAture Explorer (FAE) software suggested a candidate one-feature model according to the "one-standard error" rule. $\mathbf{c}$ The contribution of features in the final model components routinely acquired with high accuracy of 0.793 to 0.859 .

Previous studies have shown that ADC value of malignant sinonasal lesions was significantly lower than that of benign lesions [10, 22-25] as major parts of malignant tumors were composed of high cellularity whereas ADC is inversely correlated with tissue cellularity. Consistent with this notion, Sumi et al. reported lymphomas had smaller ADCs than did well-differentiated SCC nodes in the neck [26]. Maeda et al. found a statistically significant difference in ADC values between SCCs and lymphomas in the head and neck as lymphoma cells have relatively high nuclear-to-cytoplasm ratios and are densely packed [27]. Some other studies reported ADC values of poorly differentiated and undifferentiated carcinomas were significantly lower than those of moderately differentiated and well-differentiated carcinomas of the pharynx and nasal and paranasal sinus [9, 28]. In addition, ADC levels of SRCMTs were lower than of non-SRCMTs, reflecting their cellular characteristics (undifferentiated cells with high 
a

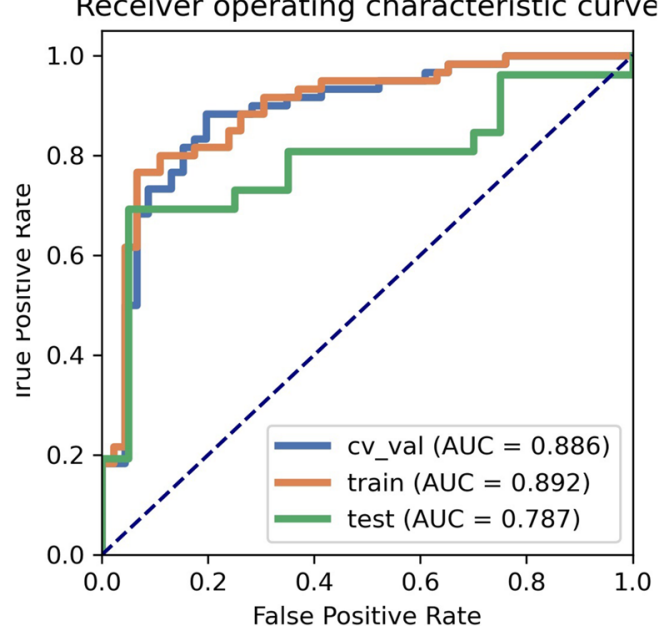

b

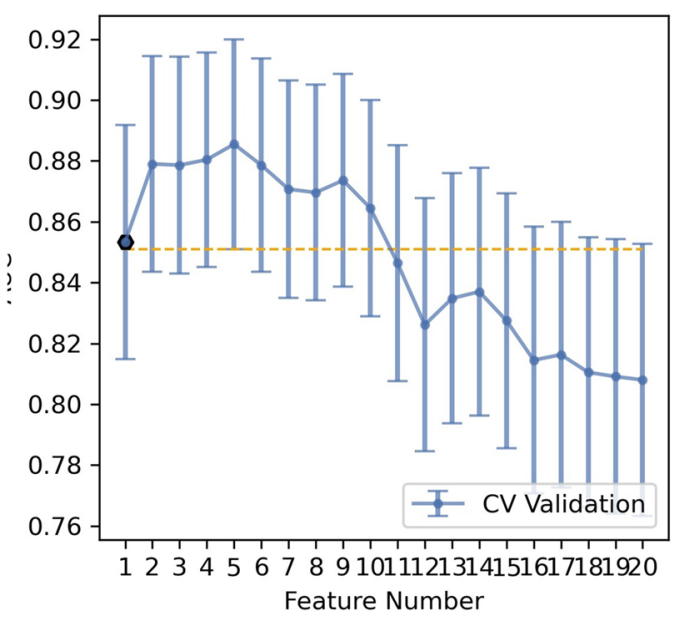

c

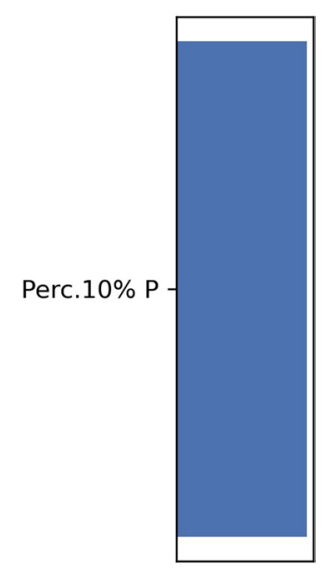

Fig. 5 Performance of model generated by relief. a Receiver operating characteristic (ROC) curves of this model using different datasets. b FeAture Explorer (FAE) software suggested a candidate one-feature model according to the "one-standard error" rule. c The contribution of features in the final model a

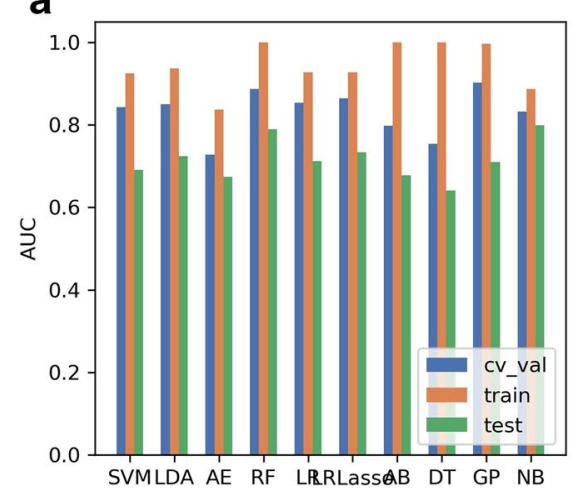

b

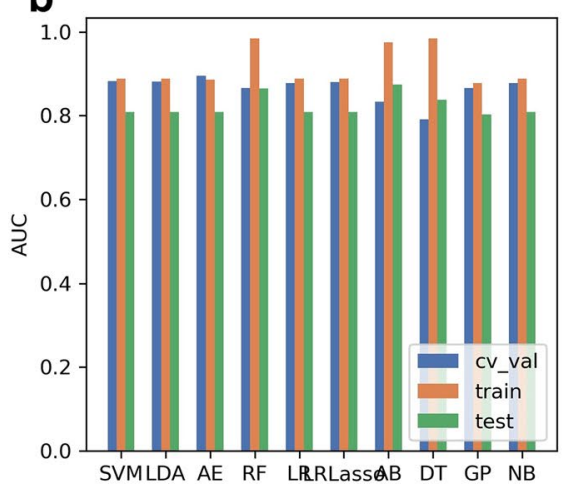

C

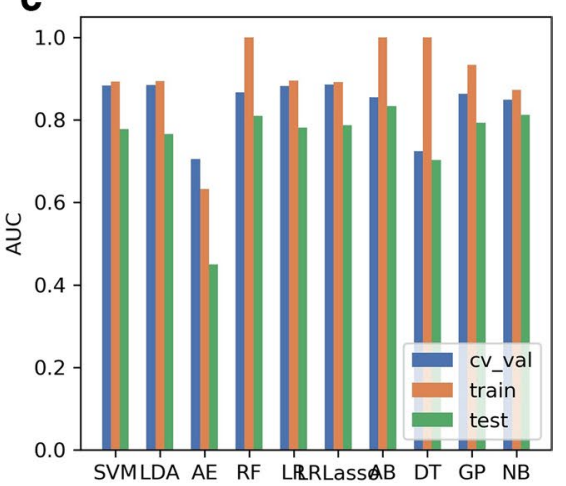

Fig. 6 Areas under the curve (AUCs) looking at different datasets. Feature selections using (a) recursive feature elimination (RFE), (b) analysis of variance (ANOVA), and (c) relief

cell attenuation, relatively small-sized nuclei, and scant cytoplasm) [8, 29]. In this study, the common parameter used to differentiate SRCMTs from non-SRCMTs was Perc. $10 \%$ in the three feature selection methods. GLCM and wavelets were the other two parameters using RFE feature selection to differentiate them. These parameters were firstorder, second-order, and higher order statistics which were described as the distribution of individual voxel values, statistical interrelationships between voxels with similar or dissimilar contrast values, and texture frequency component data extracted from the energy computed within channels, respectively. This indicates that histogram-based ADC parameters are more sensitive to histopathological features in sinonasal malignant tumors. We suspect that the cause of the efficacy of Perc.10\% ADC lies in that it reflects the complex intratumoral microstructures and heterogeneity in the whole tumor, taking a hypoxic lesion around the tiny necrotic tissue, for example.

There would always be some features which are less important on sample sets. The least important features are pruned from current set of features. RFE feature selection addresses the problem by automatically removing these features. That procedure is recursively repeated on the pruned set until the desired number of features to select is eventually reached. GP classification is a nonparametric method based on the Laplace approximation and is used for approximating the non-Gaussian posterior using the Gaussian method. It can easily handle various problems, such as an insufficient capacity for the classical linear method, complex data types, and the curse of dimensions [30]. In this study, we used an RFE feature selection and GP classifier in a LOOCV loop to boost their performance on very high-dimensional datasets, 
Table 3 The optimal area under the receiver operator characteristics curve (AUC), 95\% confidence interval (CI), standard error, accuracy, Youden index, sensitivity, specificity, accuracy, positive predictive

\begin{tabular}{|c|c|c|c|c|c|c|c|c|c|}
\hline Feature set & AUC & $95 \%$ CIs & Std & Acc & Youden Index & Sen & Spe & PPV & NPV \\
\hline Zscore_PCC_ANOVA_1_AE & 0.895 & {$[0.8260-0.9533]$} & 0.033 & 0.840 & 0.513 & 0.833 & 0.848 & 0.877 & 0.796 \\
\hline Zscore_PCC_ANOVA_3_LDA & 0.891 & {$[0.8212-0.9481]$} & 0.032 & 0.840 & 0.367 & 0.850 & 0.826 & 0.864 & 0.809 \\
\hline Zscore_PCC_ANOVA_3_LRLasso & 0.887 & [0.8114-0.9512] & 0.035 & 0.849 & 0.359 & 0.883 & 0.804 & 0.855 & 0.841 \\
\hline Zscore_PCC_ANOVA_8_SVM & 0.885 & {$[0.8100-0.9461]$} & 0.034 & 0.830 & 0.586 & 0.767 & 0.913 & 0.920 & 0.750 \\
\hline Zscore_PCC_ANOVA_3_LR & 0.884 & [0.8103-0.9449] & 0.035 & 0.840 & 0.388 & 0.883 & 0.783 & 0.841 & 0.837 \\
\hline Zscore_PCC_ANOVA_1_NB & 0.878 & [0.8025-0.9444] & 0.036 & 0.840 & 0.403 & 0.833 & 0.848 & 0.877 & 0.796 \\
\hline Zscore_PCC_ANOVA_3_RF & 0.878 & [0.7977-0.9457] & 0.038 & 0.859 & 0.520 & 0.867 & 0.848 & 0.881 & 0.830 \\
\hline Zscore_PCC_ANOVA_4_GP & 0.869 & [0.7940-0.9343] & 0.036 & 0.821 & 0.482 & 0.817 & 0.826 & 0.860 & 0.776 \\
\hline Zscore_PCC_ANOVA_3_AB & 0.865 & [0.7867-0.9337] & 0.038 & 0.802 & 0.529 & 0.683 & 0.957 & 0.954 & 0.698 \\
\hline Zscore_PCC_ANOVA_5_DT & 0.811 & [0.7298-0.8842] & 0.039 & 0.811 & 1.000 & 0.817 & 0.804 & 0.845 & 0.771 \\
\hline Zscore_PCC_RFE_10_GP & 0.902 & [0.8379-0.9519] & 0.029 & 0.830 & 0.498 & 0.867 & 0.783 & 0.839 & 0.818 \\
\hline Zscore_PCC_RFE_1_AE & 0.895 & [0.8260-0.9533] & 0.033 & 0.840 & 0.513 & 0.833 & 0.848 & 0.877 & 0.796 \\
\hline Zscore_PCC_RFE_8_RF & 0.894 & {$[0.8251-0.9525]$} & 0.033 & 0.849 & 0.565 & 0.833 & 0.870 & 0.893 & 0.800 \\
\hline Zscore_PCC_RFE_3_LDA & 0.891 & [0.8208-0.9478] & 0.032 & 0.840 & 0.367 & 0.850 & 0.826 & 0.864 & 0.809 \\
\hline Zscore_PCC_RFE_3_LRLasso & 0.886 & {$[0.8107-0.9502]$} & 0.035 & 0.849 & 0.359 & 0.883 & 0.804 & 0.855 & 0.841 \\
\hline Zscore_PCC_RFE_1_SVM & 0.883 & [0.8073-0.9460] & 0.035 & 0.821 & 0.672 & 0.733 & 0.935 & 0.936 & 0.729 \\
\hline Zscore_PCC_RFE_3_LR & 0.883 & [0.8096-0.9449] & 0.035 & 0.840 & 0.388 & 0.883 & 0.783 & 0.841 & 0.837 \\
\hline Zscore_PCC_RFE_1_NB & 0.878 & [0.8025-0.9444] & 0.036 & 0.840 & 0.403 & 0.833 & 0.848 & 0.877 & 0.796 \\
\hline Zscore_PCC_RFE_3_AB & 0.865 & [0.7867-0.9337] & 0.038 & 0.802 & 0.529 & 0.683 & 0.957 & 0.954 & 0.698 \\
\hline Zscore_PCC_RFE_9_DT & 0.808 & [0.7304-0.8795] & 0.039 & 0.811 & 1.000 & 0.833 & 0.783 & 0.833 & 0.783 \\
\hline Zscore_PCC_Relief_5_LRLasso & 0.886 & [0.8108-0.9483] & 0.035 & 0.849 & 0.359 & 0.883 & 0.804 & 0.855 & 0.841 \\
\hline Zscore_PCC_Relief_5_LDA & 0.884 & [0.8088-0.9435] & 0.033 & 0.840 & 0.332 & 0.883 & 0.783 & 0.841 & 0.837 \\
\hline Zscore_PCC_Relief_5_SVM & 0.883 & [0.8077-0.9454] & 0.035 & 0.830 & 0.511 & 0.817 & 0.848 & 0.875 & 0.780 \\
\hline Zscore_PCC_Relief_5_LR & 0.882 & [0.8055-0.9454] & 0.035 & 0.830 & 0.417 & 0.850 & 0.804 & 0.850 & 0.804 \\
\hline Zscore_PCC_Relief_3_GP & 0.880 & [0.8054-0.9423] & 0.035 & 0.840 & 0.552 & 0.800 & 0.891 & 0.906 & 0.774 \\
\hline Zscore_PCC_Relief_3_NB & 0.875 & [0.7950-0.9423] & 0.037 & 0.830 & 0.362 & 0.817 & 0.848 & 0.875 & 0.780 \\
\hline Zscore_PCC_Relief_2_AE & 0.871 & [0.7907-0.9373] & 0.037 & 0.821 & 0.441 & 0.817 & 0.826 & 0.860 & 0.776 \\
\hline Zscore_PCC_Relief_19_RF & 0.869 & [0.7947-0.9347] & 0.035 & 0.821 & 0.645 & 0.767 & 0.891 & 0.902 & 0.746 \\
\hline Zscore_PCC_Relief_5_AB & 0.855 & [0.7652-0.9254] & 0.040 & 0.821 & 0.513 & 0.767 & 0.891 & 0.902 & 0.746 \\
\hline Zscore_PCC_Relief_9_DT & 0.786 & [0.7069-0.8678] & 0.041 & 0.793 & 1.000 & 0.833 & 0.739 & 0.807 & 0.773 \\
\hline
\end{tabular}

$S V M$, support vector machine; $L D A$, linear discriminant analysis; $A E$, auto-encoder; $R F$, random forests; $L R$, logistic regression; $L R L a s s o, \operatorname{logis}-$ tic regression via Lasso; $A B$, ada-boost; $D T$, decision tree; $G P$, Gaussian process; $N B$, naive Bayes

achieving a 0.830 accuracy, 0.867 sensitivity, 0.783 specificity, and 0.902 AUC.

Our study has limitations. First, as the SRCMTs studied were of various histologic types, subgroup analyses in more details should be performed in future studies after obtaining a larger sample size and a careful consideration of the study groups. Second, as the texture-analyzing software analyzed only two-dimensional images, three-dimensional analyses which can better reflect the texture features of the entire tumor would be one of the directions of our future research. Finally, as only ADC maps were chosen, in our further studies, we will propose a multiparametric MRI investigation including ADC, T2-weighted MRI, and value (PPV), and negative predictive value (NPV) of all algorithm classifications with leave-one-out cross-validation 
Funding This study has received funding by the National Natural Science Foundation of China (no. 8193000682).

\section{Declarations}

Guarantor The scientific guarantor of this publication is Prof. Fabao Gao.

Conflict of interest One of the co-authors (X.Z.) is the employee of Siemens Healthineers (Shanghai, China). No other potential conflict of interest relevant to this article has been reported.

Statistics and biometry Fabao Gao, $\mathrm{PhD}$, kindly provided statistical advice for this manuscript.

Informed consent Written informed consent was waived by the Institutional Review Board.

Ethical approval Institutional Review Board approval was obtained.

\author{
Methodology \\ - retrospective \\ - diagnostic study \\ - performed at one institution
}

Open Access This article is licensed under a Creative Commons Attribution 4.0 International License, which permits use, sharing, adaptation, distribution and reproduction in any medium or format, as long as you give appropriate credit to the original author(s) and the source, provide a link to the Creative Commons licence, and indicate if changes were made. The images or other third party material in this article are included in the article's Creative Commons licence, unless indicated otherwise in a credit line to the material. If material is not included in the article's Creative Commons licence and your intended use is not permitted by statutory regulation or exceeds the permitted use, you will need to obtain permission directly from the copyright holder. To view a copy of this licence, visit http://creativecommons.org/licenses/by/4.0/.

\section{References}

1. Weber AL, Stanton AC (1984) Malignant tumors of the paranasal sinuses: radiologic, clinical, and histopathologic evaluation of 200 cases. Head Neck Surg 6(3):761-776

2. Silverberg E (1970) Grant R N (1970) Cancer statistics. CA Cancer J Clin 20(1):11-23

3. Simons SA, Bridge JA, Leon ME (2016) Sinonasal small round blue cell tumors: an approach to diagnosis. Semin Diagn Pathol 33(2):91-103

4. Thompson LD (2017) Small round blue cell tumors of the sinonasal tract: a differential diagnosis approach. Mod Pathol 30(s1):S1-S26

5. Ozturk K, Gencturk M, Caicedo-Granados E et al (2020) Positron emission computed tomography and magnetic resonance imaging features of sinonasal small round blue cell tumors. Neuroradiol J 33(1):48-56

6. Gencturk M, Ozturk K, Caicedo-Granados E et al (2019) Application of diffusion-weighted MR imaging with ADC measurement for distinguishing between the histopathological types of sinonasal neoplasms. Clin Imaging 55:76-82

7. Wang X, Zhang Z, Chen Q et al (2014) Effectiveness of 3 T PROPELLER DUO diffusion-weighted MRI in differentiating sinonasal lymphomas and carcinomas. Clin Radiol 69(11):1149-1156
8. Sasaki M, Sumi M, Eida S et al (2011) Multiparametric MR imaging of sinonasal diseases: time-signal intensity curve- and apparent diffusion coefficient-based differentiation between benign and malignant lesions. AJNR Am J Neuroradiol 32(11):2154-2159

9. Razek AA, Sieza S, Maha B (2009) Assessment of nasal and paranasal sinus masses by diffusion-weighted MR imaging. J Neuroradiol 36(4):206-211

10. White ML, Zhang Y, Robinson RA (2006) Evaluating tumors and tumorlike lesions of the nasal cavity, the paranasal sinuses, and the adjacent skull base with diffusion-weighted MRI. J Comput Assist Tomogr 30(3):490-495

11. Fetit AE, Novak J, Peet AC et al (2015) Three-dimensional textural features of conventional MRI improve diagnostic classification of childhood brain tumours. NMR Biomed 28(9):1174-1184

12. Villanueva-Meyer JE, Chang P, Lupo JM et al (2019) Machine learning in neurooncology imaging: from study request to diagnosis and treatment. AJR Am J Roentgenol 212(1):52-56

13. Senders JT, Zaki MM, Karhade AV et al (2018) An introduction and overview of machine learning in neurosurgical care. Acta Neurochir (Wien) 160(1):29-38

14. Collewet G, Strzelecki M, Mariette F (2004) Influence of MRI acquisition protocols and image intensity normalization methods on texture classification. Magn Reson Imaging 22(1):81-91

15. Castellano G, Bonilha L, Li LM et al (2004) Texture analysis of medical images. Clin Radiol 59(12):1061-1069

16 Haralick R, Shanmugam K, Dinstein I (1973) Textural features for image classification. IEEE Trans Syst Man Cybern SMC 3:610-621

17. Galloway M (1975) Texture analysis using gray level run lengths. Comput Graphics Image Process 4(2):172-179

18. Orphanidou-Vlachou E, Vlachos N, Davies NP et al (2014) Texture analysis of T1 - and T2 -weighted MR images and use of probabilistic neural network to discriminate posterior fossa tumours in children. NMR Biomed 27(6):632-639

19. Benesty J, Chen J, Huang Y et al (2009) Pearson correlation coefficient. Noise Reduction in Speech Processing: Springer p.1-4

20. Kira K, Rendell LA (1992) A practical approach to feature selection. Machine Learning Proceedings: Elsevier p.249-256

21. Song Y, Zhang J, Zhang YD et al (2020) FeAture Explorer (FAE): a tool for developing and comparing radiomics models. PLoS One 15(8):e237587

22. Wang P, Tang Z, Xiao Z et al (2021) Dual-energy CT in differentiating benign sinonasal lesions from malignant ones: comparison with simulated single-energy CT, conventional MRI, and DWI. Eur Radiol. https://doi.org/10.1007/s00330-021-08159-3

23. Daga R, Kumar J, Pradhan G et al (2021) Differentiation of benign from malignant sinonasal masses using diffusion weighted imaging and dynamic contrast enhanced magnetic resonance imaging. Am J Rhinol Allergy. https://doi.org/10.1177/194589242110406 02

24. Wang F, Sha Y, Zhao M et al (2017) High-resolution diffusionweighted imaging improves the diagnostic accuracy of dynamic contrast-enhanced sinonasal magnetic resonance imaging. J Comput Assist Tomogr 41(2):199-205

25. Sasaki M, Eida S, Sumi M et al (2011) Apparent diffusion coefficient mapping for sinonasal diseases: differentiation of benign and malignant lesions. AJNR Am J euroradiol 32(6):1100-1106

26. Sumi M, Nakamura T (2009) Diagnostic importance of focal defects in the apparent diffusion coefficient-based differentiation between lymphoma and squamous cell carcinoma nodes in the neck. Eur Radiol 19(4):975-981

27. Maeda M, Kato H, Sakuma $\mathrm{H}$ et al (2005) Usefulness of the apparent diffusion coefficient in line scan diffusion-weighted imaging for distinguishing between squamous cell carcinomas 
and malignant lymphomas of the head and neck. AJNR Am J Neuroradiol 26(5):1186-1192

28. Sumi M, Ichikawa Y, Nakamura T (2007) Diagnostic ability of apparent diffusion coefficients for lymphomas and carcinomas in the pharynx. Eur Radiol 17(10):2631-2637

29. Bridge JA, Bowen JM, Smith RB (2010) The small round blue cell tumors of the sinonasal area. Head Neck Pathol 4(1):84-93
30. Maniruzzaman M, Jahanur RM, Ahammed B et al (2019) Statistical characterization and classification of colon microarray gene expression data using multiple machine learning paradigms. Comput Methods Programs Biomed 176:173-193

Publisher's note Springer Nature remains neutral with regard to jurisdictional claims in published maps and institutional affiliations. 